\title{
Chlamydia and the cytoskeleton
}

The obligate intracellular pathogen Chlamydia trachomatis establishes an effective replicative niche in host cells within a membrane-bound inclusion, and to do so requires extensive interactions with host cell membrane trafficking pathways and with the cytoskeleton. Now, two recent papers have uncovered new interactions between $C$. trachomatis and the host cell cytoskeleton.

It was known that, early in the C. trachomatis developmental cycle, the inclusion is associated with microtubules, and that at the mid and late stages of development, the microtubules surrounding the inclusion show evidence of remodelling. $\mathrm{Al}-Z e e r$ et al. investigated the specific properties of the microtubules surrounding the inclusions that develop in C. trachomatis-infected Hep2 cells using immunofluorescence with antibodies specific for detyrosinated microtubules. Detyrosinated microtubules were much more abundant in infected cells than in uninfected cells and were clearly associated with the inclusion. Treatment of C. trachomatis-infected HeLa cells with inhibitors of detyrosinated microtubule formation led to a dosedependent decrease in the formation of infectious progeny. Additionally, detyrosinated microtubules were found to be more resistant to treatment with the microtubule inhibitor nocodazole than non-modified microtubules, indicating this posttranslational modification confers increased stability.

Previous work had also shown that, in C. trachomatis-infected cells, the Golgi is fragmented into smaller Golgi stacks. In this study, the authors found that almost half of all the inclusions in the C. trachomatisinfected cells they examined were associated with both detyrosinated microtubules and Golgi stacks. After 1 hour of nocodazole treatment, the Golgi stacks dispersed from the inclusions and, when the nocodazole was washed out, the microtubules repolymerized and the Golgi stacks redistributed to the inclusions. As the association with the Golgi stacks is thought to have a key role in

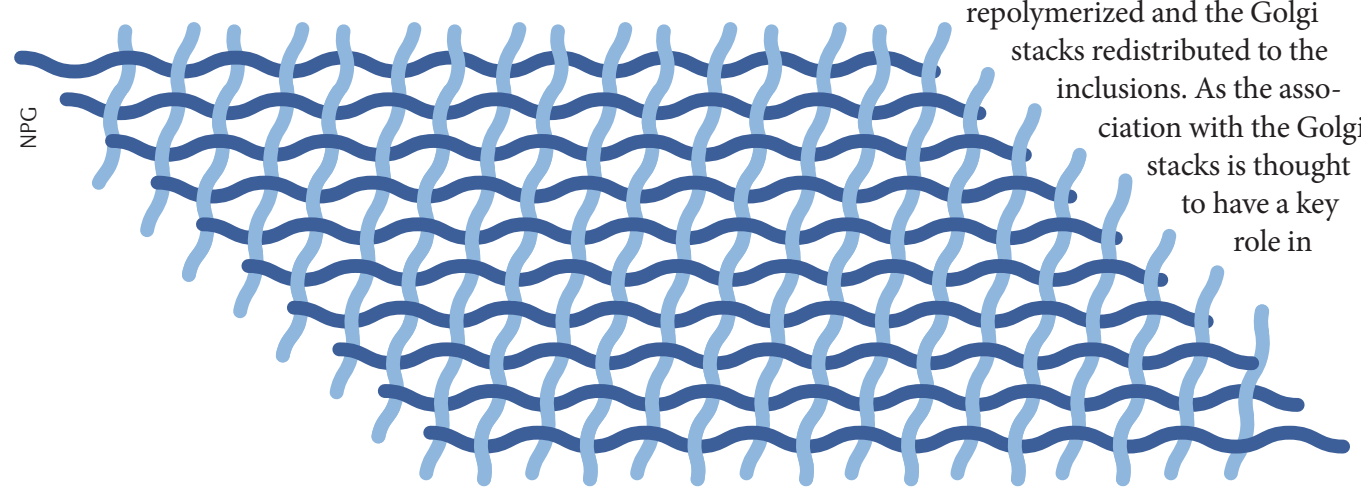

Chlamydia nutrient acquisition, the authors propose that the detyrosinated, stabilized microtubules provide the physical support necessary for the Golgi stacks to fulfil their role in nutrient supply.

In addition to microtubules, previous work had also shown that Chlamydia inclusions are associated with actin and with intermediate filaments, but the involvement of septins had not been investigated. Using immunofluorescence, Volceanova et al. found that septin 2, septin 9 , septin 11 , and probably also septin 7 , form a fibrillar structure around C. trachomatis inclusions in HeLa cells, and co-staining assays showed that the septins colocalized with actin. RNA interference revealed that the septins are required for the organization of the actin coat around the inclusion. Although the authors found no evidence that the heteromeric septin-actin fibres were involved in vesicular trafficking to the inclusion, they did find that they were required for extrusion of the inclusion from host cells at the end of the developmental cycle.

Sheilagh Molloy

ORIGINAL RESEARCH PAPERS Al-Zeer, M. A et al. Chlamydia trachomatis remodels stable microtubules to coordinate Golgi stack

recruitment to the chlamydial inclusion surface. Mol. Microbiol. http://dx.doi.org/10.1111/ mmi.12829 (2014) | Volceanova, L. et al. Septins arrange $\mathrm{F}$-actin-containing fibers on the Chlamydia trachomatis inclusion and are required for normal release of the inclusion by extrusion. mBio http://dx.doi.org/10.1128/mBio.01802-14 (2014) 